\title{
Microfluidic Tensiometry Technique for the Characterization of the Interfacial Tension between Immiscible Liquids
}

\author{
Lawrence W. Honaker, Jan P. F. Lagerwall,* and V. S. R. Jampani*(-) \\ Experimental Soft Matter Physics Group, University of Luxembourg, L-1511 Luxembourg, Grand Duchy of Luxembourg
}

Supporting Information

ABSTRACT: The interfacial tension between two immiscible fluids is of critical importance for understanding many natural phenomena as well as in industrial production processes; however, it can be challenging to measure this parameter with high accuracy. Most commonly used techniques have significant shortcomings because of their reliance on other data such as density or viscosity. To overcome these issues, we devise a technique that works with very small sample quantities and does not require any data about either fluid, based on micropipette aspiration techniques. The method facilitates the generation of a droplet of one fluid inside of the other, followed by immediate in situ aspiration of the droplet into a constricted channel. A modified Young-Laplace equation is then used to relate the pressure needed to produce a given deformation of the droplet's radius to the interfacial tension. We demonstrate this technique on different systems with interfacial tensions ranging from sub-millinewton per meter to several hundred millinewton per meter, thus over 4 orders of magnitude, obtaining precise results in agreement with the literature solely from experimental observations of the droplet deformation.

\section{INTRODUCTION}

The interfacial tension between fluids is a property that governs many processes, ranging from natural phenomena such as the locomotion of insects on water, ${ }^{1}$ the superhydrophobicity of lotus leaves (the "lotus effect"), ${ }^{2}$ respiration, ${ }^{3}$ and fluid flow instability ${ }^{4}$ to wetting and capillary forces that influence many industrial applications, for instance, in emulsion technology (important in foods, pharmaceuticals, cosmetics, etc.) and composites preparation. ${ }^{5}$ To determine the interfacial tension between two immiscible liquids, a wide range of techniques exist. Plate and ring methods monitor the wetting of a surface by a liquid, ${ }^{5}$ and drop shape analysis techniques focus on the curvature of a known droplet, such as pendant and sessile drops. $^{6-10}$ Bubble methods observe the deformation of a shell around an air pocket ${ }^{11}$ or the pressure necessary to form air bubbles of known radii, relating that pressure to the surface or interfacial tension by the Young-Laplace equation. ${ }^{12}$ More recently, microfluidics techniques have been employed to determine the interfacial tension on microscopic scales by observing the dynamics of droplet deformations in a flow, ${ }^{13-21}$ using a minimum of fluid while still obtaining precise data.

These techniques have proven themselves to be powerful and versatile; however, they also have significant shortcomings. In particular, they either are only applicable for certain combinations of fluids or require precise knowledge of additional material parameters, such as the liquid densities or viscosities. ${ }^{6,8,14}$ If such data are not available with high accuracy, or if the values of the required parameter for the two liquids are very similar, large uncertainties arise in the established interfacial tension value. ${ }^{9}$ Moreover, many techniques require relatively large volumes of the liquids to be studied.

Other techniques for measuring interfacial tension include aspiration methods, initially applied to determine the cortical tension of living animalian cells or lipid membranes. ${ }^{22-24}$ These techniques use carefully tuned pressure differences to aspirate material into a micropipette, the pressure needed to aspirate the cell being related to the unaspirated and aspirated radii from a modified form of the Young-Laplace equation which relates the interfacial tension, $\gamma$, to the pressure difference $\delta P$ necessary to induce aspiration compared to the pressure for a stationary undeformed cell, and the radii of curvature of the unaspirated and the aspirated cell, $R_{1}$ and $R_{2}$, respectively ${ }^{22-28}$

$$
\delta P=2 \gamma\left(\frac{1}{R_{2}}-\frac{1}{R_{1}}\right)
$$

Aspiration techniques, however, were used on cells and membranes with fixed and defined boundaries and not for fluids in general. To overcome this and other issues, here we present a microfluidic tensiometry technique that generates droplets before aspirating them back into a constricted channel and measuring the interfacial tension in situ. The pressure needed to aspirate the cell or droplet is linearly related to the interfacial tension by eq 1 , requiring only the radii of curvature of the unaspirated and the aspirated droplets to obtain the interfacial

Received: October 7, 2017

Revised: December 10, 2017

Published: January 24, 2018 
tension. The interfacial tension values obtained in our lab are compared to previously obtained literature data, demonstrating that our new tensiometry technique works for a broad range of combinations of immiscible liquids, including those with very low interfacial tensions, on the order of sub-millinewton per meter, and those for which present techniques have great difficulties in producing precise and reliable data. ${ }^{9,24}$ Measurements as a function of time can also produce dynamic interfacial tension data with high resolution.

\section{EXPERIMENTAL SECTION}

Materials. TWEEN 20 (poly(oxyethylene) (20) sorbitan monolaurate, 99\%, Sigma-Aldrich), Span 80 (sorbitan oleate, 99\%, SigmaAldrich), hexadecane (>99\%, Sigma-Aldrich), 5CB (4-cyano-4'pentylbiphenyl, $>99 \%$, Xinhua Chemical Company), DMOAP (dimethyloctadecyl[3(trimethoxy-silyl)propyl]ammonium chloride, $42 \%$ in methanol, Sigma-Aldrich), galinstan (Smart Elements $\mathrm{GmbH}$ ), PETA (pentaerythritol tetraacrylate, 99\%, Sigma-Aldrich), and sodium hydroxide ( $>99 \%$, Sigma-Aldrich) were all used as received without further purification. Ultrapure deionized water $(0.055$ $\mu \mathrm{S} / \mathrm{cm}$ ) was obtained from a Sartorius arium water purifier.

Device Fabrication. The devices used were based on techniques for glass capillary flow-focusing devices presented by Utada et al. ${ }^{29}$ In short, capillaries for the injection of the dispersed phase were fabricated from round borosilicate glass capillaries $(1.0 \mathrm{~mm}$ outer diameter, Drummond) pulled using a Sutter P-100 pipette puller to varying degrees of taper and cut with a Narishige microforge or cut by hand and smoothed with a 1000 grit sandpaper to the desired orifice diameters. Capillaries for collecting the produced droplets were fabricated from the same capillaries used for the injection of the dispersed phase, either used as supplied or after the creation of a constriction at a point in the capillary using the pipette puller. The capillaries were then cleaned with oxygen plasma. When working with galinstan as a dispersed phase and water as an outer phase, no further treatments of the glass surfaces were performed, as plasma treatment alone creates hydrophilic substrates. The glass capillaries were then stored under ultrapure deionized water prior to device assembly. When water or $5 \mathrm{CB}$ was used as the dispersed phase, the capillaries were then treated with a dilute DMOAP solution to prevent wetting of the dispersed phase and dried in a vacuum oven at $110{ }^{\circ} \mathrm{C}$ for at least 30 min before being assembled into devices.

Each device was then assembled on a clean microscope slide, using a square glass capillary (VitroTubes, $1.0 \mathrm{~mm}$ inner diameter) to encase the injection and collection capillaries. A Sterican 21 gauge cannula was cut to accommodate the injection point for the outer phase fluid. The capillaries and the cannula were positioned under a microscope and fixed in place with a Pattex Super Mix Metal two-part epoxy. When the injection and collection capillaries were properly positioned and fixed in place, transparent UV-reactive epoxy (Norland NO86 optical adhesive) was filled by capillary action into the empty space of the channel in a manner such that enough space was left for the outer phase to flow into the channel; the process is shown in Figure 1.



Figure 1. Process of filling UV glue for sealing around the injection capillary. The UV glue (blue-gray in the online figure) is filled into the square capillary up to the dashed line before sealing.
The device was then cured under a Wood's lamp for $10 \mathrm{~min}$, solidifying the UV adhesive. Schematics of the assembled devices are shown in Figure 2.



Figure 2. Schematics of the microfluidic devices used. (a) Device used for measuring interfacial tension at the tip of the injection capillary. (b) Device used for measuring interfacial tension at a constriction.

Alternating rinses of reagent-grade isopropanol and ultrapure water were then flushed through each of the capillaries to both clean the device of aqueous and organic residues and to ensure no leaking was present prior to each device's use in an experiment.

Method. A Fluigent MFCS-EZ pressure pump system equipped with two 1034 mbar pressure channels, one 345 mbar channel, and one 25 mbar channel was used to flow the liquids into the device through tubing by pressurizing septum-equipped vials containing the liquids to be studied. This instrument integrates both a rapid-response pressure pump and continuous pressure monitoring. When materials not sensitive to air were used, droplets were generated by flowing the continuous and dispersed phases under various pressures, producing droplets of different sizes by tuning the ratio of the flow rates between the fluids. A syringe pump was instead used to flow air-sensitive liquids, such as galinstan, to prevent the material from oxidizing. ${ }^{30}$

After droplets of the dispersed phase were generated from an injection capillary into a collection capillary, depending on the device used, they were then aspirated, by applying pressure from the exit of the channel, either into the channel from which the droplets were produced, as demonstrated in Figure 3, or into a constriction created further down the collection tube, as shown in Figure 4.

Images of the aspiration process were continuously captured using a high-resolution camera (PixeLINK M15C) and matched with the corresponding pressure at each stage of droplet deformation. The continuous monitoring of the pressure applied from the right end of the collection capillary, whether using the device shown in Figure 3 or in Figure 4, allows us to measure the change $\delta P_{1}$ that is needed to initiate aspiration of the droplet either at the orifice of the injection capillary or into the constricted region of the collection capillary, as shown in Figure 5 . Knowing $\delta P_{1}$, the corresponding measured change of the droplet radius upon aspiration from $R_{1}$ to $R_{2}$ then allows us to calculate, via eq 1 , the interfacial tension between the two immiscible liquids under investigation (see Supporting Information for interfacial tension derivation and aspiration Videos S1 and S2). The use of a constricted geometry allows us to produce different degrees of aspiration with different applied pressures.

\section{RESULTS}

Isotropic Fluids. We first validated our devices with aqueous solutions of TWEEN 20 in several concentrations as the dispersed phase and solutions of different concentrations of Span 80 in hexadecane as the continuous phase. These solutions were previously extensively characterized by Hashimoto et al. by pendant drop techniques ${ }^{31}$ and present a wide range of potential interfacial tensions by simple variation of the 

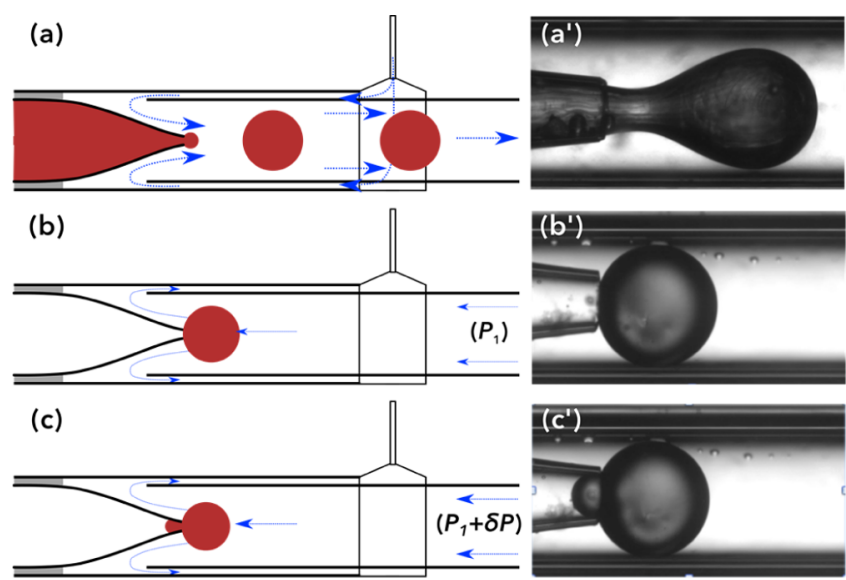

Figure 3. Schematic and corresponding experimental images (here, $5 \mathrm{CB}$ in a $1.0 \mathrm{mM}$ CTAB solution) of droplet production and aspiration back into the injection channel. In the schematic diagrams, blue arrows represent the flow direction. $\left(a / a^{\prime}\right)$ Schematic representation of the droplet production process; $\left(b / b^{\prime}\right)$ droplet held stationary in the channel before aspiration into the injection channel, with a pressure $P_{1}$ applied from the exit of the collection channel; and $\left(\mathrm{c} / \mathrm{c}^{\prime}\right)$ aspiration of the droplet into the injection channel with a pressure $P_{1}+\delta P$ applied from the exit of the collection channel.

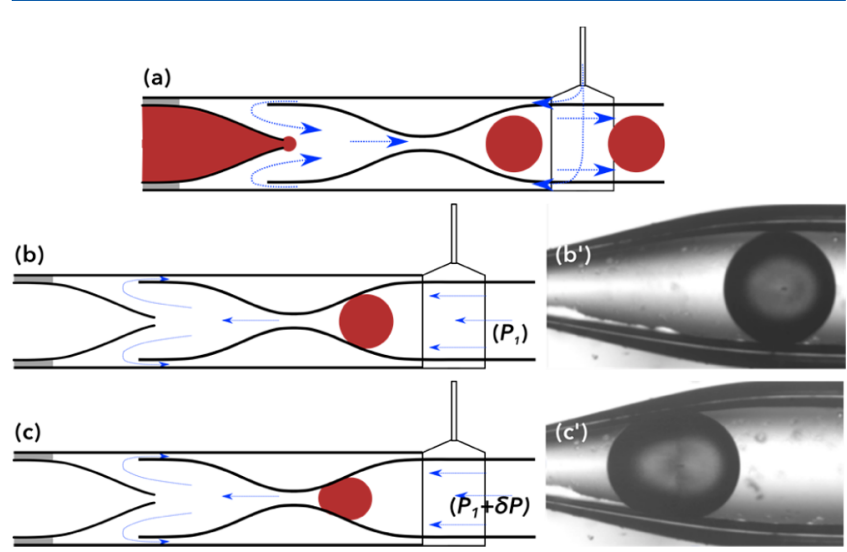

Figure 4. Schematic and corresponding experimental images (here, of $5 \mathrm{CB}$ in a $1.0 \mathrm{mM}$ CTAB solution) of droplet production and aspiration back into the injection channel. In the schematic diagrams, blue arrows represent the flow direction. (a) Schematic of the device and the droplet production process; $\left(\mathrm{b} / \mathrm{b}^{\prime}\right)$ droplet being held stationary in the collection channel just after the constriction, with pressure $P_{1}$ applied from the collection tube exit; $\left(\mathrm{c} / \mathrm{c}^{\prime}\right)$ droplet aspiration process, with a droplet deformation achieved upon applying pressure $P_{1}+\delta P$ from the collection tube exit.

concentration of the surfactant in the two phases, thus giving a good means to test our system and the technique. The results we obtained are presented and compared to the literature values in Table 1.

As can be seen, the obtained interfacial tension values are reasonably in agreement with the literature values, the main source of deviation being in the resolution with which the pressure pump allows us to adjust $P$. Lower interfacial tensions are measured with extremely small pressure differences; with the measurement uncertainty of the pressure pump used, this propagates to high relative uncertainties.

Liquid Metals. The second group of systems we tested was with a dispersed phase of galinstan, gallium alloyed with indium and tin such that it exists as a liquid at room temperature. For continuous phases, we used aqueous sodium hydroxide $(\mathrm{NaOH})$ solutions of concentrations ranging from 0.01 to 1.0 $\mathrm{M} . \mathrm{NaOH}$ solutions were chosen because strongly acidic and/ or basic conditions will remove the oxide skin that rapidly forms when the gallium alloy comes into contact with oxygen, thus allowing the liquid metal to once again behave as a liquid. ${ }^{30,32-35}$ The measured interfacial tensions are presented in Table 2.

These observations are generally in accordance with literature observations, ${ }^{35}$ and within the measurement uncertainty, they suggest that the $\mathrm{pH}$ or the concentration of $\mathrm{NaOH}$ does not play a significant role in the interfacial tension between the two phases provided that the $\mathrm{pH}$ of the solution is 10 or greater. We were unable to produce satisfactory measurements of the interfacial tension of galinstan against 1.0 M hydrochloric acid $(\mathrm{HCl})$ solution. Bilodeau et al. ${ }^{32}$ found that the pendant drop equilibration time for a droplet of galinstan in $1.0 \mathrm{M} \mathrm{HCl}$ was $1.5 \mathrm{~h}$ compared to immediate equilibration in the case of $0.1 \mathrm{M} \mathrm{NaOH}$. The inability to apply our technique to the galinstan- $\mathrm{HCl}$ solution interface is thus likely a product of hydrochloric acid less readily removing the oxide skin compared to sodium hydroxide, thus leading to a solidlike behavior at the droplet boundary. Our technique works for any liquid-liquid interface, provided that the dispersed phase droplets do not break up or wet the capillary walls. When an aqueous $\mathrm{HCl}$ solution is used as the continuous phase, the two liquids are separated by a very thin layer of solid gallium oxide that, moreover, also results in a certain effective wetting of the glass capillary walls, explaining the difficulty.

We additionally were able to measure the interfacial tension between a continuous phase of degassed PETA, a viscous (viscosity > $1000 \mathrm{mPa} \cdot \mathrm{s}$ ) acrylate monomer, and galinstan droplets, obtaining an interfacial tension of $569 \pm 15 \mathrm{mN} / \mathrm{m}$, showing that this technique can also work with exceptionally viscous systems. We know of no literature data for comparison in this case.

Anisotropic Liquids (Liquid Crystals). As a further test of our tensiometer, we decided to measure the interfacial tension between the common synthetic nematic liquid crystalline material $5 \mathrm{CB}$ and aqueous surfactant solutions. The reported density values of $5 \mathrm{CB}$ are not entirely consistent: most indicate a low density of around $1.008 \mathrm{~g} / \mathrm{mL}^{36,37}$ at room temperature, but values as high as $1.035 \mathrm{~g} / \mathrm{mL}$ have been reported. ${ }^{38}$ Such variation may raise concerns about the ability to accurately measure the interfacial tension using common techniques. ${ }^{9,10,38,39}$ Our system gives an opportunity to establish a value that is entirely independent of the reliability of the density data.

We first measured the interfacial tension between $5 \mathrm{CB}$ and an aqueous solution of $1.0 \mathrm{M} \mathrm{CTAB}$ (hexadecyltrimethylammonium bromide), giving us a value of $3.2 \pm 0.8 \mathrm{mN} / \mathrm{m}$ at room temperature, which falls within the values reported by Kim et al. ${ }^{9}$ Their reported values are in the range $1.5-5.5 \mathrm{mN} /$ $\mathrm{m}$ at room temperature, where the uncertainty was largely due to the uncertain density of $5 \mathrm{CB}$ and the limitations of the pendant drop technique used in that study.

A difficulty in working with $\mathrm{CTAB}$, however, is its high Krafft temperature: at temperatures below $23{ }^{\circ} \mathrm{C}$, the surfactant will crystallize out of the solution. Therefore, to further investigate the interfacial behavior of $5 \mathrm{CB}$, we measured the interfacial tension of $5 \mathrm{CB}$ also against aqueous solutions of SDS (sodium dodecyl sulfate). At $10 \mathrm{mM}$, above the surfactant's critical micelle concentration (CMC), reported as $\sim 8.2 \mathrm{mM}$ at $25^{\circ} \mathrm{C}$, 

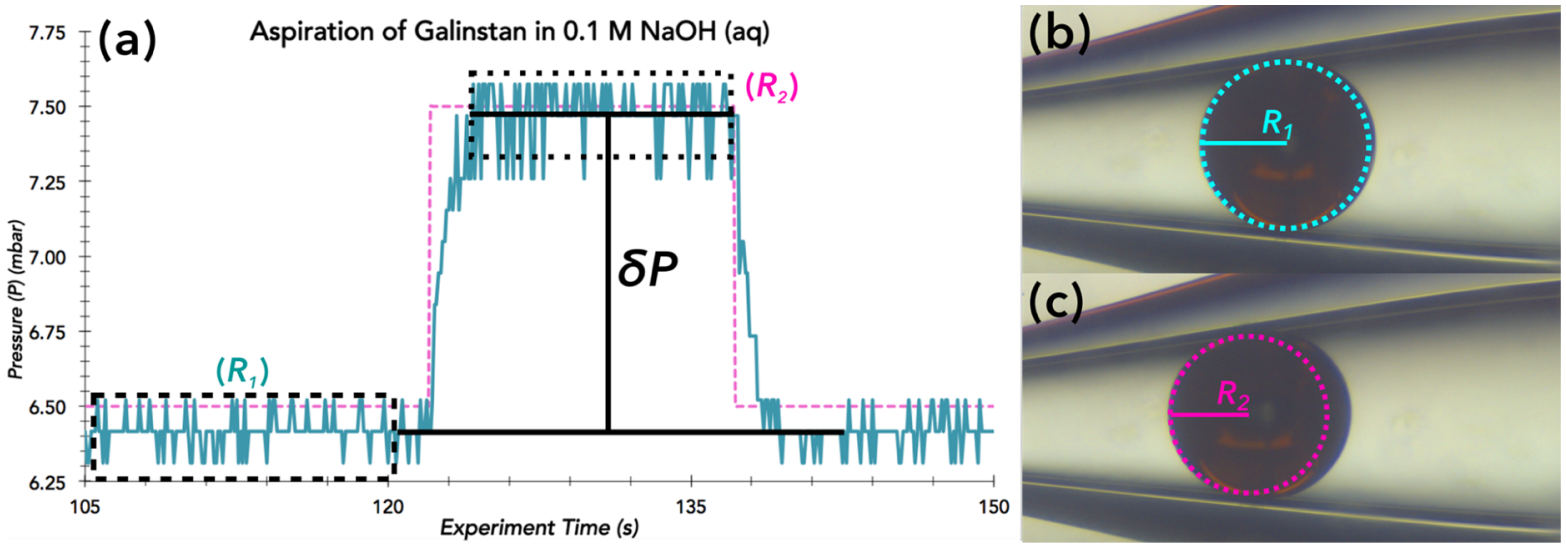

Figure 5. Demonstration of the variation of pressure $P$ applied from the exit of the collection capillary, measured during the aspiration process, and the resulting variation of droplet radius, both of which are used to determine the interfacial tension. (a) Real-time graph of $P$ as a function of time, showing the reading from the pneumatic pump before, during, and after the aspiration of the droplet into the constricted channel. (b) Initial state, when the droplet is held fixed to the right of the constricted region of the channel without any movement or visible deformation, used as the state for the initial value of $P_{1}$. (c) Aspirated state, in which the droplet has entered the constricted region of the channel thanks to an increase in $P$ to the value $P_{1}+\delta P$, with a consequent distortion of its shape. The change in pressure between the states in (b) and (c), $\delta P$, is the quantity needed in eq 1.

Table 1. Interfacial Tensions between Solutions of TWEEN 20 (aq) as the Dispersed Phase and Solutions of Span 80 in Hexadecane as the Continuous Phase Compared to Literature Values from Hashimoto et al. ${ }^{31}$ (Listed in Parentheses)

\begin{tabular}{cccc}
\multicolumn{5}{c}{ concentration TWEEN $20(\mathrm{aq}, \mathrm{w} / \mathrm{w})$} \\
concentration Span 80 in hexadecane & $0.02 \%$ & $0.2 \%$ & $2.0 \%$ \\
$(\mathrm{w} / \mathrm{w})$ & $4.4 \pm 0.5 \mathrm{mN} / \mathrm{m}(4.4 \pm 0.2 \mathrm{mN} / \mathrm{m})$ & $2.3 \pm 0.3 \mathrm{mN} / \mathrm{m}(2.5 \pm 0.2 \mathrm{mN} / \mathrm{m})$ & $2.7 \pm 0.4 \mathrm{mN} / \mathrm{m}(2.4 \pm 0.4 \mathrm{mN} / \mathrm{m})$ \\
$0.03 \%$ & $1.7 \pm 0.2 \mathrm{mN} / \mathrm{m}(1.4 \pm 0.2 \mathrm{mN} / \mathrm{m})$ & $0.8 \pm 0.3 \mathrm{mN} / \mathrm{m}(0.7 \pm 0.1 \mathrm{mN} / \mathrm{m})$ & $0.4 \pm 0.2 \mathrm{mN} / \mathrm{m}(0.2 \pm 0.0 \mathrm{mN} / \mathrm{m})$ \\
$0.3 \%$ & &
\end{tabular}

Table 2. Interfacial Tensions of Galinstan against $\mathrm{NaOH}$ Solutions and Their Standard Deviations Obtained from a Set of at Least Five Measurements

\begin{tabular}{cc} 
concentrat3ion base & mean interfacial tension $(\mathrm{mN} / \mathrm{m})$ \\
$1.0 \mathrm{M} \mathrm{NaOH}(\mathrm{pH} 14)$ & $462 \pm 32$ \\
$0.1 \mathrm{M} \mathrm{NaOH}(\mathrm{pH} \mathrm{13})$ & $446 \pm 18$ \\
$0.01 \mathrm{M} \mathrm{NaOH}(\mathrm{pH} 12)$ & $442 \pm 18$ \\
\hline
\end{tabular}

the measured interfacial tension was $3.7 \pm 0.3 \mathrm{mN} / \mathrm{m}$. This value is close to the number obtained for the $\mathrm{CTAB}$ solution of concentration above its $\mathrm{CMC}\left(\sim 0.92 \mathrm{mM}\right.$ at $25^{\circ} \mathrm{C}$ for $\left.\mathrm{CTAB}\right)$. Six further concentrations of SDS were tested, with the complete results summarized in Figure 6. At all SDS concentrations below the CMC, a logarithmic relationship of surfactant concentration to the measured equilibrium interfacial tension was observed, similar to previous observations of a smectic liquid crystal phase in contact with aqueous surfactant solutions. ${ }^{11}$

The values obtained in Figure 6 are equilibrium interfacial tension values, obtained over experimental time scales of up to $30 \mathrm{~min}$ with no change in the behavior observed. Using the constricted capillary technique, we were additionally able to obtain time-dependent dynamic interfacial tension values as the surfactant adsorbs to the water-liquid crystal interface. This is accomplished by setting a fixed aspiration pressure to be just enough to hold the droplet at the entrance to the constriction and then observing the droplet retract into the constriction as its interfacial tension decreases with time. An example of such a dynamic interfacial tension measurement is presented in Figure 7. The interfacial tension of $5 \mathrm{CB}$ decreases very quickly with time, reaching an equilibrium state within 2 to $3 \mathrm{~min}$ of droplet

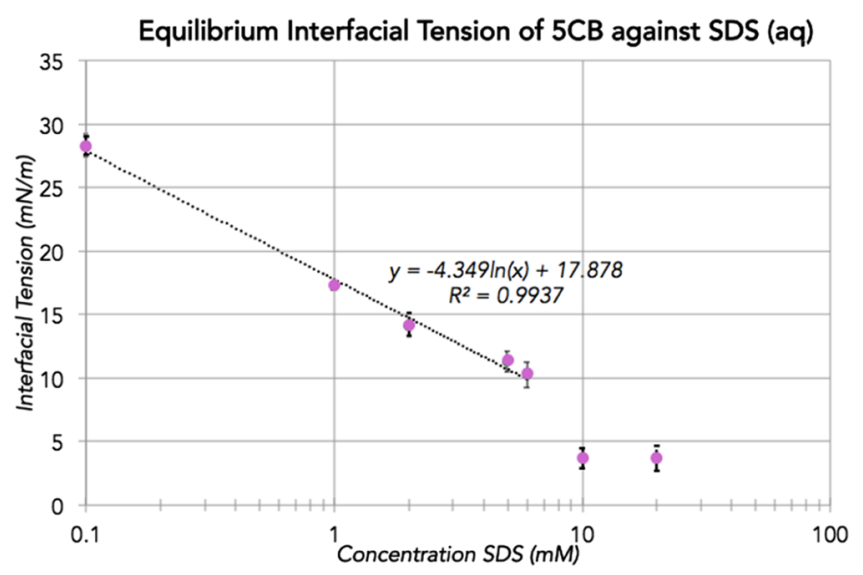

Figure 6. Equilibrium interfacial tension of $5 \mathrm{CB}$ against seven SDS (aq) solutions measured using the constricted capillary technique. The error bars represent standard deviations of a minimum of five measurements at each concentration; the logarithmic curve is fitted to the data for the sub-CMC concentrations.

production, similar to the behavior shown by Harth et al. for a smectic liquid crystal in a surfactant solution. ${ }^{11}$

We also tested our technique to see if we were able to directly measure the interfacial tension of $5 \mathrm{CB}$ against pure water, a measurement that has proven problematic to perform with traditional methods of interfacial tensiometry. We obtained as a value of $30.8 \pm 7.5 \mathrm{mN} / \mathrm{m}$, which is reasonably in concordance with the data reported by Proust et al. ${ }^{40}$ The high standard deviation in our measurements comes from the tendency of $5 \mathrm{CB}$ droplets to easily wet glass surfaces in the absence of surfactant. Some possible treatments for overcoming the wetting issues are outlined in the Supporting Information. 


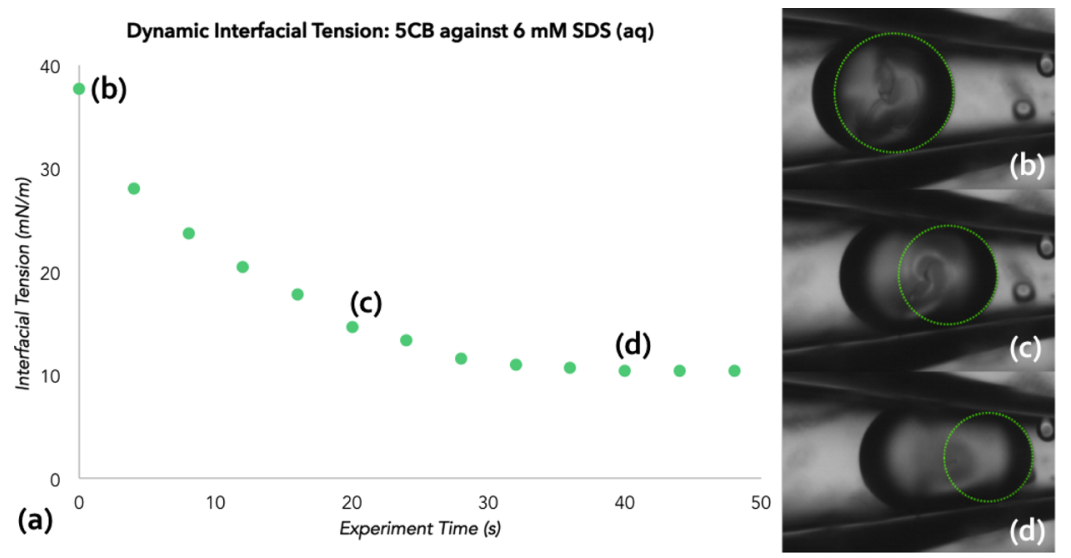

Figure 7. Dynamic interfacial tension of a droplet of $5 \mathrm{CB}$ against $6.0 \mathrm{mM}$ SDS (aq) solution, measured over $50 \mathrm{~s}$ from when the droplet was captured in the constriction. (a) Calculated interfacial tensions as a function of experimental time; (b) initial state at $t=0 \mathrm{~s}$; (c) at $t=20 \mathrm{~s}$; and (d) at $t=40 \mathrm{~s}$.

Finally, we measured the surface tension of the $6.0 \mathrm{mM}$ SDS solution by placing a small air droplet in the constriction (see Supporting Information). The measurement of the surface tension works well with our technique, producing a reasonable value of $35 \pm 4 \mathrm{mN} / \mathrm{m}$.

\section{DISCUSSION}

Although dynamic interfacial tension data were easily accessible, it was not possible to measure the interfacial tension of the droplet immediately after production because of the design of our current setup. With a more optimized design (with, e.g., the constriction placed immediately after droplet generation), it should be possible to minimize the time from droplet generation to interfacial tension measurement, thereby further extending the dynamic measurement.

Because it is essential that the dispersed phase does not wet the capillary walls, adequate surface treatment is required. This is easy to accomplish when the two materials used are, for example, a nonpolar alkane and an aqueous solution: treatment to prevent wetting of the dispersed phase will ensure wetting of the continuous phase, such as the use of DMOAP (hydrophobic treatment) with an alkane as the continuous phase and an aqueous solution as the dispersed phase (as presented above). When either or both of the liquids have some amphiphilic character, however, such as with liquid crystal droplets in water, this becomes considerably more challenging. Although most liquid crystals, for example, do not wet to hydrophobic-treated substrates well, they wet to them better than an aqueous solution wets. The issue is then a question of finding a substrate to which the dispersed phase wets less than the continuous phase does.

Wetting issues become less of a concern when a high concentration of surfactant is used, as the surfactant effectively adsorbs to the dispersed phase interface and creates a layer that reduces adhesion to the glass capillaries: this is normally best achieved at concentrations above the CMC for a given surfactant, but an absence of wetting can also be observed for certain surfactant concentrations below the CMC. Ultimately, preventing wetting can be difficult to achieve for some combinations of liquids, possibly presenting a more fundamental challenge for our technique. However, for most such difficult liquid combinations, an alternative technique for measuring the interfacial tension will typically fail.

\section{CONCLUSIONS}

In summary, we have demonstrated a microfluidics technique for tensiometry that applies to a wide range of systems of immiscible liquids covering a large range of orders of interfacial tensions, even when nothing is known about their further properties such as density or viscosity. It requires very small amounts of liquid and is thus ideal for studying the interfacial tension between precious liquids available only in small quantities, for example, custom-synthesized liquids or a naturally occurring liquid in scarce quantities. The main general limitation is equipment-related, namely, the resolution of the pressure pump used: many of the measured pressure changes $\delta P$, particularly for the $5 \mathrm{CB}$-water systems, were on the order of $0.1 \mathrm{mbar}$; with a minimum resolution of $0.01 \mathrm{mbar}$ for the pressure pump, non-negligible uncertainty results. Importantly, this uncertainty is not intrinsic to the technique and can be reduced significantly by tailoring of the equipment. Assuming adequate pressure resolution and substrates successfully treated to prevent wetting of the dispersed phase, our new technique can precisely measure interfacial tensions from as little as a few micronewton per meter to the order of newton per meter.

\section{ASSOCIATED CONTENT}

\section{Supporting Information}

The Supporting Information is available free of charge on the ACS Publications website at DOI: 10.1021/acs.langmuir.7b03494.

Derivation of the modified Young-Laplace equation, measurement of the surface tension of $6.0 \mathrm{mM}$ SDS solution, and suggested wetting treatments for various combinations of liquids (PDF)

Aspiration of Galinstan in $0.1 \mathrm{M} \mathrm{NaOH}$ solution (AVI) Aspiration of $5 \mathrm{CB}$ in $0.01 \mathrm{M}$ SDS solution (AVI)

\section{AUTHOR INFORMATION}

\section{Corresponding Authors}

*E-mail: jan.lagerwall@lcsoftmatter.com (J.P.F.L.).

*E-mail: softmatter@vsrjampani.com (V.S.R.J.).

ORCID $\odot$

V. S. R. Jampani: 0000-0003-0881-2065 


\section{Author Contributions}

V.S.R.J. devised the experimental methods and technique. V.S.R.J. and L.W.H. carried out the experiments. V.S.R.J., L.W.H., and J.P.F.L. analyzed and interpreted the data. All authors contributed to preparing the manuscript.

\section{Funding}

This work was funded in part by an Aide à la formation-recherche (AFR) grant from the Luxembourg National Research Foundation (project code LIMEFLOW) and by a Consolidator Grant from the European Research Council (project code INTERACT, ERC grant agreement no. 648763).

\section{Notes}

The authors declare no competing financial interest.

\section{ACKNOWLEDGMENTS}

The authors would like to thank Catherine Reyes for helpful suggestions and Angelo Manna for technical support.

\section{REFERENCES}

(1) Andersen, N. M.; Cheng, L. The Marine Insect Halobates (Heteroptera: Gerridae): Biology, Adaptations, Distribution, and Phylogeny. In Oceanography and Marine Biology; CRC Press, 2004; pp 119-179.

(2) Neinhuis, C.; Barthlott, W. Characterization and Distribution of Water-repellent, Self-cleaning Plant Surfaces. Ann. Bot. 1997, 79, 667677.

(3) Fathi-Azarbayjani, A.; Jouyban, A. Surface tension in human pathophysiology and its application as a medical diagnostic tool. BioImpacts 2015, 5, 29-44.

(4) Driessen, T.; Jeurissen, R.; Wijshoff, H.; Toschi, F.; Lohse, D. Stability of viscous long liquid filaments. Phys. Fluids 2013, 25, 062109.

(5) du Noüy, P. L. An interfacial tensiometer for universal use. J. Gen. Physiol. 1925, 7, 625-631.

(6) Boyce, J. F.; Schürch, S.; Rotenberg, Y.; Neumann, A. W. The Measurement of Surface and Interfacial Tension by the Axisymmetric Drop Technique. Colloids Surf. 1984, 9, 307-317.

(7) del Río, O. I.; Neumann, A. W. Axisymmetric drop shape analysis: computational methods for the measurement of interfacial properties from the shape and dimensions of pendant and sessile drops. J. Colloid Interface Sci. 1997, 196, 136-147.

(8) Saad, S. M. I.; Policova, Z.; Neumann, A. W. Design and accuracy of pendant drop methods for surface tension measurement. Colloids Surf., A 2011, 384, 442-452.

(9) Kim, J.-W.; Kim, H.; Lee, M.; Magda, J. J. Interfacial tension of a nematic liquid crystal/water interface with homeotropic surface alignment. Langmuir 2004, 20, 8110-8113.

(10) Popov, P.; Honaker, L. W.; Mirheydari, M.; Mann, E. K.; Jákli, A. Chiral nematic liquid crystal microlenses. Sci. Rep. 2017, 7, 1603.

(11) Harth, K.; Shepherd, L. M.; Honaker, J.; Stannarius, R. Dynamic interface tension of a smectic liquid crystal in anionic surfactant solutions. Phys. Chem. Chem. Phys. 2015, 17, 26198-26206.

(12) Mysels, K. J. The Maximum Bubble Pressure Method of Measuring Surface Tension, Revisited. Colloids Surf. 1990, 43, 241262.

(13) Nguyen, N. T.; Lassemono, S.; Chollet, F. A.; Yang, C. Microfluidic Sensor for Interfacial Tension Measurement and Method for Measuring Interfacial Tension. U.S. Patent 7975531 B2, 2011

(14) Cabral, J. T.; Hudson, S. D. Microfluidic approach for rapid multicomponent interfacial tensiometry. Lab Chip 2006, 6, 427.

(15) Nguyen, N.-T.; Lassemono, S.; Chollet, F. A.; Yang, C. Microfluidic Sensor for Dynamic Surface Tension Measurement. IEE Proc.-Nanobiotechnol. 2006, 153, 102-106.

(16) Hudson, S. D.; Cabral, J. T.; Goodrum, W. J.; Beers, K. L.; Amis, E. J. Microfluidic interfacial tensiometry. Appl. Phys. Lett. 2005, 87, 081905 .
(17) Xu, J. H.; Li, S. W.; Lan, W. J.; Luo, G. S. Microfluidic Approach for Rapid Interfacial Tension Measurement. Langmuir 2008, 24, $11287-11292$.

(18) Wang, K.; Lu, Y. C.; Xu, J. H.; Luo, G. S. Determination of dynamic interfacial tension and its effect on droplet formation in the T-shaped microdispersion process. Langmuir 2009, 25, 2153-2158.

(19) Steegmans, M. L. J.; Warmerdam, A.; Schroën, K. G. P. H.; Boom, R. M. Dynamic interfacial tension measurements with microfluidic Y-junctions. Langmuir 2009, 25, 9751-9758.

(20) Moiré, M.; Peysson, Y.; Pannacci, N.; Herzhaft, B.; Colin, A.; Dalmazzone, C. A New Microfluidic Tensiometer for Optimizing EOR Formulations. SPE Improved Oil Recovery Conference, 2016; pp 1-14.

(21) Brosseau, Q.; Vrignon, J.; Baret, J.-C. Microfluidic Dynamic Interfacial Tensiometry ( $\mu$ DIT). Soft Matter 2014, 10, 3066-3076.

(22) Hochmuth, R. M. Micropipette aspiration of living cells. J. Biomech. 2000, 33, 15-22.

(23) Lee, S.; Kim, D. H.; Needham, D. Equilibrium and Dynamic Interfacial Tension Measurements at Microscopic Interfaces Using a Micropipet Technique. 1. A New Method for Determination of Interfacial Tension. Langmuir 2001, 17, 5537-5543.

(24) Lee, S.; Kim, D. H.; Needham, D. Equilibrium and Dynamic Interfacial Tension Measurements at Microscopic Interfaces Using a Micropipet Technique. 2. Dynamics of Phospholipid Monolayer Formation and Equilibrium Tensions at the Water-Air Interface. Langmuir 2001, 17, 5544-5550.

(25) Derganc, J.; Božič, B.; Svetina, S.; Žekš, B. Stability Analysis of Micropipette Aspiration of Neutrophils. Biophys. J. 2000, 79, 153-162.

(26) Fung, Y.-C. Biomechanics: Mechanical Properties of Living Tissue, 2nd ed.; Springer New York: New York, 1993.

(27) Lee, L. M.; Liu, A. P. The Application of Micropipette Aspiration in Molecular Mechanics of Single Cells. J. Nanotechnol. Eng. Med. 2014, 5, 040902.

(28) Molino, D.; Quignard, S.; Gruget, C.; Pincet, F.; Chen, Y.; Piel, M.; Fattaccioli, J. On-Chip Quantitative Measurement of Mechanical Stresses During Cell Migration with Emulsion Droplets. Sci. Rep. 2016, 6, 29113.

(29) Utada, A. S.; Lorenceau, E.; Link, D. R.; Kaplan, P. D.; Stone, H. A.; Weitz, D. A. Monodisperse Double Emulsions Generated from a Microcapillary Device. Science 2005, 308, 537-541.

(30) Liu, T.; Sen, P.; Kim, C.-J. Characterization of nontoxic liquidmetal alloy galinstan for applications in microdevices. J. Microelectromech. Syst. 2012, 21, 443-450.

(31) Hashimoto, M.; Garstecki, P.; Stone, H. A.; Whitesides, G. M. Interfacial instabilities in a microfluidic Hele-Shaw cell. Soft Matter 2008, 4, 1403.

(32) Bilodeau, R. A.; Zemlyanov, D. Y.; Kramer, R. K. Liquid Metal Switches for Environmentally Responsive Electronics. Adv. Mater. Interfaces 2017, 4, 1600913.

(33) Khan, M. R.; Trlica, C.; So, J.-H.; Valeri, M.; Dickey, M. D. Influence of Water on the Interfacial Behavior of Gallium Liquid Metal Alloys. ACS Appl. Mater. Interfaces 2014, 6, 22467-22473.

(34) Dickey, M. D. Emerging applications of liquid metals featuring surface oxides. ACS Appl. Mater. Interfaces 2014, 6, 18369-18379.

(35) Gol, B.; Tovar-Lopez, F. J.; Kurdzinski, M. E.; Tang, S.-Y.; Petersen, P.; Mitchell, A.; Khoshmanesh, K. Continuous transfer of liquid metal droplets across a fluid-fluid interface within an integrated microfluidic chip. Lab Chip 2015, 15, 2476-2485.

(36) Gannon, M. G. J.; Faber, T. E. The surface tension of nematic liquid crystals. Philos. Mag. A 1978, 37, 117-135.

(37) Oweimreen, G. A.; Shihab, A. K.; Halhouli, K.; Sikander, S. F. Density Measurements in the Nematic and Isotropic Phases of $5 \mathrm{CB}$ and Dilute Solutions of Tetraethylmethane in 5CB. Mol. Cryst. Liq. Cryst. 1986, 138, 327-338.

(38) Tintaru, M.; Moldovan, R.; Beica, T.; Frunza, S. Surface tension of some liquid crystals in the cyanobiphenyl series. Liq. Cryst. 2001, 28, 793-797.

(39) Rai, P. K.; Denn, M. M.; Maldarelli, C. Interfacial Tension of Liquid Crystalline Droplets. Langmuir 2003, 19, 7370-7373. 
(40) Proust, J. E.; Perez, E.; Ter-Minassian-Saraga, L. Films minces de cristal liquide nématique sur support liquide Structure, tensions superficielles et tension de ligne. Colloid Polym. Sci. 1978, 256, 666681. 